\title{
Model predictive control: a passive scheme
}

\author{
P. Falugi * \\ * EEE Department, Imperial College, London, SW7 2AZ, UK. (e-mail: \\ p.falug@imperial.ac.uk).
}

\begin{abstract}
This note studies the formulation of model predictive control exploiting passivity properties. The introduction of passive constraints in model predictive control schemes is particularly appealing since robustness against model uncertainty is inherently guaranteed. The potential of the discussed control scheme is shown on the regulation problem of a robot manipulator.
\end{abstract}

\section{INTRODUCTION}

Model predictive control (MPC) is a widely used design technique for multi-input multi-output systems in many practical applications since input and state constraints can be taken into account in the design problem directly Mayne et al. [2000], Qin and Badgwell [2003]. In the vast majority of MPC formulations the stability of the closed loop system is based on the decrease of the optimal cost which is usually obtained by an appropriate choice of the terminal ingredients as surveyed in Mayne et al. [2000], Rawlings and Mayne [2009], Limon et al. [2009]. Essentially, all the techniques employed to prove stability of the MPC controller make use of Lyapunov functions. The relationship between optimal control, nonlinear MPC and control Lyapunov function is discussed in Primbs et al. [2000] where a control Lyapunov function is introduced as additional constraint in the receding horizon scheme. Here we are interested in controlling passive systems optimizing prescribed performance while satisfying input and state constraints. The notion of passivity is a very important concept in many areas and in particular in electrical, electronic and mechanical engineering where the systems are modeled using Euler-Lagrange equations Ortega et al. [1998]. Passivity is, roughly speaking, a restatement of energy conservation principles. In many applications, the question whether a system is passive or not can be answered from physical considerations on the way the system interacts with its environment. Passivity is characterized by the property that at any time the amount of energy the system can possibly supply to its environment can not exceed the amount of energy that has been supplied to it. In other words, when time evolves, a passive system absorbs a fraction of its supplied energy and transforms it in another form. When a system is passive it can be advantageous to use the information on the physical structure of the system and design a controller to shape the energy of the system and even change how energy flows inside the system. The main idea is to design a controller, called passivity based control (PBC) Ortega and Spong [1989], such that the closed-loop system is again a passive system. There is a multitude of applications making use of passivity based controllers such as walking robots, bilateral teleoperators, pendular systems massbalance systems, inventory control, reactors, power systems, power converters, motors, magnetic levitation systems, underwater vehicles, surface vessels, spacecrafts, formation control, synchronization, consensus problems just to mention a few. It is well know Van der Schaft [2000] that the negative interconnection of two passive systems is still a passive system. Using the concept of passivity the controlled system can be made robust to model uncertainties For these reasons we are interested in the problem of designing optimal passive controllers for passive nonlinear systems affected by constraints. Note that the passivity of the nonlinear plant is only required with respect to a fictitious output and such fictitious output always exists as long as a control Lyapunov function exists. In this paper we introduce in the MPC formulation a constraint imposing the passivity of the controller and we study the stability and robustness properties of the closed loop system. A passivity based MPC without input and state constraints has been proposed in Raff et al. [2007] for the first time where the aim was to impose passivity of the closed loop systems without the use of terminal ingredients. In Yan et al. [2013] the robustness against model discrepancy of the passivity based MPC scheme introduced in Raff et al. [2007] has been discussed in terms of the truncated $\mathcal{L}_{2}$ norm adding a dissipative term in the passivity constraint. Here instead we face the problem of implicitly designing a passive controller using MPC and taking into account input and state constraints.

\section{PROBLEM FORMULATION}

The system to be controlled is described by

$$
\begin{aligned}
& \dot{x}=f(x, u) \\
& y=h(x)
\end{aligned}
$$

where $x \in \mathbb{R}^{n}$ is the state of the system, $u \in \mathbb{R}^{m}$ is the control input and $y \in \mathbb{R}^{m}$ the output. The control $u$ and state $x$ are required to satisfy the hard constraints $u \in \mathbb{U}$ and $x \in \mathbb{X}$ where $\mathbb{U}$ and $\mathbb{X}$ are compact. In order to guarantee existence and uniqueness of the solution it is assumed that $f(\cdot)$ is locally Lipschitz continuous in $\mathbb{X}$, i.e. for each compact subset $\mathcal{C} \subset \mathbb{X}$ there exist a constant $L_{\mathcal{C}}$ such that $\|f(x, u)-f(\hat{x}, u)\| \leq L_{\mathcal{C}}\|x-\hat{x}\|$ for all $x, \hat{x} \in \mathcal{C}$ and $u \in \mathbb{U}$. Moreover $h(\cdot)$ is continuous. Without loss of generality, we consider the regulation problem to the equilibrium pair $(\bar{x}, \bar{u})=(0,0) \subset \mathbb{X} \times \mathbb{U}$ while satisfying input and state constraints. 
Definition 1. The system (1) is strictly output passive if there exists a positive semidefinite storage function $S(x)$ and $\rho>0$ such that

$$
\int_{t_{0}}^{t_{f}}\left(u^{\prime}(\tau) y(\tau)-\rho y^{\prime}(\tau) y(\tau)\right) d \tau \geq S\left(x\left(t_{f}\right)\right)-S\left(x\left(t_{0}\right)\right)
$$

$\forall x\left(t_{0}\right) \in \mathbb{X}$ and $u(\cdot) \in \mathcal{U}$ where $\mathcal{U}$ denotes the space of measurable and locally essentially bounded functions with values in $\mathbb{U}$.

Note that, in general, it is possible to adopt the more general notion of strict input and output passivity as described in Teel et al. [2010]

$$
\begin{aligned}
\int_{t_{0}}^{t_{f}}\left(u^{\prime}(\tau) y(\tau)-y^{\prime}(\tau) \phi(y(\tau))-u^{\prime}(\tau) \psi(u(\tau))\right) d \tau \\
\quad \geq S\left(x\left(t_{f}\right)\right)-S\left(x\left(t_{0}\right)\right)
\end{aligned}
$$

where $y^{\prime} \phi(y)>0$ and $u^{\prime} \psi>0$ for all $y \neq 0, u \neq 0$. However in this paper, for sake of clarity, we consider strictly output passive systems with $\phi=\rho y$.

Equivalently, system (1) is strictly output passive if there exists a constant $\beta_{x_{0}} \geq 0$, depending on $x\left(t_{0}\right)$, such that

$$
\int_{t_{0}}^{t}\left(u^{\prime}(\tau) y(\tau)-\rho y^{\prime}(\tau) y(\tau)\right) d \tau \geq-\beta_{x_{0}} \quad \forall t \geq t_{0}
$$

and all functions $u(\cdot) \in \mathcal{U}$.

A controlled system enjoys strong robustness properties with respect to model uncertainties preserving passivity of the open-loop system. In fact, a closed loop system is strictly output passive if it is given by a negative feedback interconnection of two strictly output passive systems as illustrated in fig. 1. Moreover if a system is strictly output

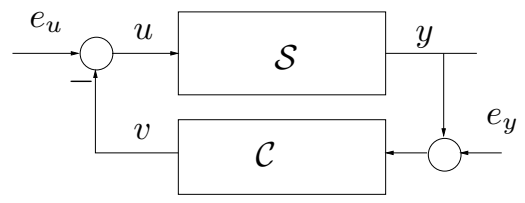

Fig. 1. System interconnection

passive then it has finite $\mathcal{L}_{2}$ gain (see i.e Van der Schaft [2000], R. Sepulchre and Kokotović [1997]. It is possible to compute a strictly output feedback system $\mathcal{C}$ (see fig. 1) with output $v=-u$ using MPC with the additional integral constraint

$$
\int_{t_{0}}^{t_{f}}\left(-u^{\prime}(\tau) y(\tau)-\gamma u^{\prime}(\tau) u(\tau)\right) d \tau \geq-\beta
$$

where $\beta \geq 0$ and $\gamma>0$ are constants that need to be selected using the knowledge of the system and the set $\mathbb{X}$. The constraint (4) can be included in the MPC formulation introducing an additional state variable $z$ as follows

$$
\dot{z}=u^{\prime} y+\gamma u^{\prime} u
$$

with boundary constraints $z\left(t_{0}\right)=z_{0}, z\left(t_{f}\right) \leq \beta$, where $z_{0}=0$ at time $t_{0}=0$ and at $t_{0}>0$ it depends on the solution to the optimization problem at the previous time instant as discussed in detail in the next section. Similar constraints taking into account average performance in Economic Model Predictive Control have been used in Angeli et al. [2012].

\section{PASSIVE MODEL PREDICTIVE CONTROL}

Given the passive system defined in (1) the proposed model predictive controller using the passivity condition (4) is obtained at each time instant as the solution to the optimization problem $\mathbb{P}(x, z)$

$$
\begin{aligned}
V(x, z)= & \min _{u(\cdot)} \int_{0}^{T} \ell(x(\tau), u(\tau)) d \tau \\
& \text { subject to } \\
& \dot{x}=f(x, u) \quad x(0)=x \\
& y=h(x) \\
& \dot{z}=u^{\prime} y+\gamma u^{\prime} u \quad z(0)=z \\
& u(\tau) \in \mathbb{U}, x(\tau) \in \mathbb{X}, z(\tau) \leq \beta \quad \tau \in[0, T] \\
& x(T)=0
\end{aligned}
$$

where $\beta$ is the positive constant introduced in (4), the stage cost $\ell(\cdot)$ is a positive definite function and satisfies $\ell(0,0)=0$. If the current state of the composite system at time $t$ is $(x, z)$ we denote the optimal solution to $\mathbb{P}(x, z)$ as $\left(x^{0}(\cdot ; x, z), u^{0}(\cdot ; x, z), z^{0}(\cdot ; x, z), y^{0}(\cdot ; x, z)\right)$. The control applied to the system is

$$
\kappa(x, z) \triangleq u^{0}(\tau ; x, z) \quad \tau \in[0, \delta]
$$

in the interval $[t, t+\delta]$; at time $t+\delta$ the optimal control problem is solved again given the initial states $x(0)=$ $x^{0}(t+\delta ; x, z)$ and $z(0)=z^{0}(t+\delta ; x, z)$. The non negative constants $\beta$ and $\gamma$ can be pre-assigned or obtained, at the first time instant, as the solution to the optimization problem $\mathbb{P}^{0}(x)$ where $\beta$ and $\gamma$ are decision variables as well

$$
\begin{aligned}
V^{*}(x)= & \inf _{u(\cdot), \beta, \gamma} \int_{0}^{T} \ell(x(\tau), u(\tau)) d \tau \\
& \text { subject to } \\
& \dot{x}=f(x, u) \quad x(0)=x \\
& y=h(x) \\
& u(\tau) \in \mathbb{U}, x(\tau) \in \mathbb{X} z(\tau) \leq \beta \quad \tau \in[0, T] \\
& \dot{z}=u^{\prime} y+\gamma u^{\prime} u \quad z(0)=0 \\
& x(T)=0 \quad \beta>0 \quad \gamma>0
\end{aligned}
$$

Remark 1. The terminal constraint $x(T)=0$ has been chosen for simplicity. Since the system (1) can be stabilized with a feedback $u=-g(y)$ it is possible to use as terminal constraint a set $X_{f} \subseteq \mathbb{X}$ that is positive invariant for the system $\dot{x}=f(x,-g(\bar{y}))$ and satisfies $-g(h(x)) \in \mathbb{U}$ for all $x \in X_{f}$.

\section{STABILITY AND ROBUSTNESS}

To establish convergence of the nominal closed loop system to the zero equilibrium we use, as is customary, Lyapunov arguments. Let $x\left(t ; x_{0}, 0\right)$ denote the solution to $\dot{x}=$ $f(x, \kappa(x, z))$ at time $t$ if $x_{0}=x(0)$ and $\kappa(x, z)$ is given by (7). Also, let $\mathcal{X} \triangleq\{x \in \mathbb{X} \mid \mathbb{P}(x, 0)$ is feasible $\}$ i.e. the set $\mathcal{X}$ denotes the set of initial states $x$ for which a solution to $\mathbb{P}(x, 0)$ exists.

Theorem 1. Under the above assumptions, every solution of $\dot{x}=f(x, \kappa(x, z))$ with initial state $x \in \mathcal{X}$, where $\kappa(x, z)$ is solution to the optimization problem $\mathbb{P}(x, z)$, remains in $\mathcal{X}$ and converges to the zero equilibrium state.

Proof: - The proof is by induction, showing that feasibility at time $t$ implies feasibility at time $t+\delta$.

Let assume that $\left(x^{0}(\tau ; x, z, t), u^{0}(\tau ; x, z, t), z^{0}(\tau ; x, z, t)\right.$, 
$\left.y^{0}(\tau ; x, z, t)\right), \tau \in[0, T]$, is the optimal solution at time $t$ to $\mathbb{P}(x, z)$. Now we prove that at time $t+\delta$ the input function

$$
\tilde{u}(\tau)= \begin{cases}u^{0}(\tau+\delta ; x, z, t) & \tau \in[0, T-\delta] \\ 0 & \tau \in[T-\delta, T]\end{cases}
$$

is a feasible solution to $\mathbb{P}\left(x^{0}(\delta ; x, z, t), z^{0}(\delta ; x, z, t)\right)$. Let

$$
\tilde{x}(\tau)= \begin{cases}x^{0}(\tau+\delta ; x, z, t) & \tau \in[0, T-\delta] \\ 0 & \tau \in[T-\delta, T]\end{cases}
$$

By construction, the pointwise-in-time constraints $\tilde{x}(\tau) \in$ $\mathbb{X}, \tilde{u}(\tau) \in \mathbb{U}$ and the terminal condition $\tilde{x}(T)=0$ are satisfied. Let assume that at time $t$

$$
z(T)=z(0)+\int_{0}^{T}\left(u^{\prime}{ }^{\prime}(\tau) y^{0}(\tau)+\gamma u^{\prime 0}(\tau) u^{0}(\tau)\right) d \tau \leq \beta
$$

is satisfied where $z(0)$ is the initial condition at time $t$. At time $t+\delta$ we have that

$$
\begin{aligned}
& \tilde{z}(T) \triangleq \tilde{z}(0)+\int_{0}^{T}\left(\tilde{u}^{\prime}(\tau) h(\tilde{x}(\tau))+\gamma \tilde{u}^{\prime}(\tau) \tilde{u}(\tau)\right) d \tau \\
& =\tilde{z}(0)+\int_{\delta}^{T}\left(u^{\prime 0}(\tau) y^{0}(\tau)+\gamma u^{\prime 0}(\tau) u^{0}(\tau)\right) d \tau
\end{aligned}
$$

where $\tilde{z}(0)=z(\delta)$ and $\tilde{z}(\cdot)$ describes the evolution of $z(\cdot)$ when the feasible solution (9) is employed. Adding and subtracting the term $\int_{0}^{\delta}\left(u^{\prime} 0(\tau) y^{0}(\tau)+\gamma u^{0}(\tau) u^{0}(\tau)\right) d \tau$ to the equation (11) we obtain

$$
\begin{aligned}
& \tilde{z}(T)=\tilde{z}(0)-\int_{0}^{\delta}\left(u^{\prime} 0(\tau) y^{0}(\tau)+\gamma u^{\prime 0}(\tau) u^{0}(\tau)\right) d \tau \\
& +\int_{0}^{T}\left(u^{\prime 0}(\tau) y^{0}(\tau)+\gamma u^{\prime 0}(\tau) u^{0}(\tau)\right) d \tau
\end{aligned}
$$

Since, by definition, $\int_{0}^{\delta}\left(u^{\prime}(\tau) y^{0}(\tau)+\gamma u^{\prime}(\tau) u^{0}(\tau)\right) d \tau=$ $z(\delta)-z(0)$ and $\tilde{z}(0)=z(\delta)$ we obtain $\tilde{z}(T) \leq \beta$ which shows feasibility of the integral constraint; feasibility at any time ensures the invariance of the set $\mathcal{X}$. Now we prove the convergence of $x(t ; x, z, 0)$ to the zero state equilibrium; The optimal value function $V(x, z)$ is selected as a candidate Lyapunov function. It satisfies $V(x, z) \geq 0$ and $V(x, z)=0$ if and only if $x=0 \forall z \in \mathbb{R}^{+}$. For all $x \in \mathcal{X}$ there exist $\mathcal{K}_{\infty}$ functions $\alpha_{1}(\cdot)$ and $\alpha_{2}(\cdot)$ such that

$$
\alpha_{1}(\|x\|) \leq V(x, z) \leq \alpha_{2}(\|x\|) \quad \forall z \in \mathbb{R}^{+}
$$

since $\ell(\cdot)$ is a positive definite function and satisfies $\ell(0,0)=0$. At time $t$ given $x$ the optimization problem $\mathbb{P}(x, z)$ is solved and the control $u^{0}(\tau ; x, z, t), \tau \in[0, \delta]$ is applied to the system. After the sampling time $\delta$ the optimization problem $\mathbb{P}\left(x_{\delta}, z_{\delta}\right)$ is solved where $x_{\delta} \triangleq x(t+$ $\delta ; x, z, t)$. Since the control sequence $\tilde{u}(\cdot)$ is feasible but not necessarily optimal for $\mathbb{P}\left(x_{\delta}, z_{\delta}\right)$, the following relation holds

$$
\begin{aligned}
& V\left(x_{\delta}, z_{\delta}\right) \leq \int_{0}^{T} \ell(\tilde{x}(\tau), \tilde{u}(\tau)) d \tau= \\
& V(x, z)-\int_{0}^{\delta} \ell\left(x^{0}(\tau), u^{0}(\tau)\right) d \tau
\end{aligned}
$$

where $\tilde{u}(\tau)$ is the feasible input defined in (9) and the functions $x^{0}(\tau ; x, z)$ are $u^{0}(\tau ; x, z)$ are the optimal solution to $\mathbb{P}(x, z)$.

Since $\int_{0}^{\delta}\left(\ell\left(x^{0}(\tau), u^{0}(\tau)\right) d \tau\right) \geq 0$ for all $x$ such that $\|x\|>$ $0,0 \leq V\left(x_{\delta}, z_{\delta}\right)<V^{0}(x, z)$ is satisfied for all $x$ such that $\|x\|>0$ and convergence of $x(t ; x, z, 0)$ to the zero state equilibrium is obtained by standard Lyapunov arguments.
The strict passivity constraint introduced in the optimization problem $\mathbb{P}(x, z)$ enforces strict passivity of the open loop controller having $v(t)=-u(t)$ as output and $y(t)$ as input. Now we are interested to show strictly passivity of the closed loop controller obtained implicitly by the MPC strategy.

Theorem 2. Let $w(t) \in h\left(\mathcal{X}_{z_{t}}\right)$ where $z_{t}$ is the value of $z(0)$ at time $t$ and $\mathcal{X}_{z_{t}} \triangleq\left\{x \in \mathbb{X} \mid \mathbb{P}\left(x, z_{t}\right)\right.$ is feasible $\}$ denotes the set of feasible states defined by $\mathbb{P}(x, z)$. The closed loop controller, defined by

$$
\begin{array}{r}
\dot{z}(\tau)=\kappa\left(x\left(t_{0}\right), z\left(t_{0}\right)\right) w(\tau)+\gamma v(\tau)^{\prime} v(\tau) \\
v(\tau)=-\kappa\left(x\left(t_{0}\right), z\left(t_{0}\right)\right) \\
t_{0} \geq 0, \tau \in\left[t_{0} ; t_{0}+\delta\right]
\end{array}
$$

is strictly output passive for all $x(0) \in \mathcal{X}$ where $z(0)=0$.

Proof: - Strict passivity of (12) is guaranteed if

$$
\int_{0}^{t}\left(-v^{\prime}(\tau) w(\tau)+\gamma v^{\prime}(\tau) v(\tau)\right) d \tau \leq \hat{\beta}_{x_{0}}
$$

for all $t \geq 0$ and $x(t) \in \mathcal{X}_{z_{t}}$ where $\hat{\beta}_{x_{0}} \geq 0$ with $x_{0} \in \mathcal{X}$ and $v(t)$ is the solution of the system (12) with $w(t) \in h\left(\mathcal{X}_{z_{t}}\right)$ and $z(0)=0$. Since $h(\cdot)$ is continuous and $\mathcal{X}_{z_{t}}$ is bounded, $w(t)$ is bounded. Moreover since $\mathbb{P}(x, z)$ is feasible at any time if it is feasible at time zero and $w(t) \in h\left(\mathcal{X}_{z_{t}}\right)$ we have

$$
\begin{aligned}
& z\left(t_{0}\right)+\int_{t_{0}}^{t_{0}+\delta}\left(\kappa(x(\tau), z(\tau))^{\prime}(w(\tau))+\right. \\
& \left.\gamma \kappa(x(\tau), z(\tau))^{\prime} \kappa(x(\tau), z(\tau))\right) d \tau+ \\
& \int_{t_{0}+\delta}^{t_{0}+T}\left(u^{\prime}(\tau) y(\tau)+\gamma u^{\prime}(\tau) u(\tau)\right) d \tau \leq \beta
\end{aligned}
$$

for all $x(t) \in \mathcal{X}_{z_{t}}$ where

$$
\begin{gathered}
z\left(t_{0}\right)=\int_{0}^{t_{0}}\left(\kappa(x(\tau), z(\tau))^{\prime}(w(\tau))+\right. \\
\gamma \kappa(x(\tau), z(\tau))^{\prime} \kappa(x(\tau), z(\tau)) d \tau
\end{gathered}
$$

and $\int_{t_{0}+\delta}^{t_{0}+T}\left(u^{\prime}(\tau) y(\tau)+\gamma u^{\prime}(\tau) u(\tau)\right) d \tau$ is given by the open loop controller and predictions. Since condition (13) holds for any $t_{0}>0$ by feasibility of $\mathbb{P}(x, z)$ at any time the system (12) is strictly output passive for all $x(t) \in \mathcal{X}_{z_{t}}$.

Robustness of the closed loop system follows from the fact that the negative feedback interconnection of strictly output passive systems is still strictly output passive. Let assume that the real system described by

$$
\begin{aligned}
& \dot{x}_{s}=\tilde{f}\left(x_{s}, u\right) \\
& y_{s}=\tilde{h}\left(x_{s}\right)
\end{aligned}
$$

is strictly output passive in $\mathcal{X}$ for some $\tilde{\beta} \geq \tilde{\beta}_{x_{0}}, \tilde{\rho} \geq \tilde{\rho}_{x_{0}}>$ 0 with $x_{0} \in \mathcal{X}$. In order to achieve robust stability we need to assume that the system (14) is zero-state detectable Van der Schaft [2000], i.e $\lim _{t \rightarrow \infty} x_{s}(t)=0$ if $u(t)=0$, $y(t)=0, \forall t \geq 0$ where $x_{s}(t)$ is the solution of system (14). Proposition 1. Let assume that the perturbed system (14) is zero-state detectable and strictly output passive and that $\mathbb{P}\left(x_{s}, z\right)$ is feasible at any time then the closed loop system

$$
\begin{aligned}
& \dot{x}_{s}=\tilde{f}\left(x_{s}, \kappa\left(x_{s}, z\right)\right) \\
& \dot{z}=\kappa(x, z) w+\gamma \kappa(x, z) \kappa(x, z)
\end{aligned}
$$

where $\kappa(x, z)$ is solution to $\mathbb{P}\left(x_{s}, z\right)$, satisfies $\lim _{t \rightarrow \infty} x_{s}(t)=$ 0 .

Proof: - Since the system (14) is strictly output passive there exists a storage function $S_{s}(x)$ and $\tilde{\rho}>0$ such that 


$$
\int_{t_{0}}^{t_{f}}\left(u^{\prime}(\tau) y(\tau)-\tilde{\rho} y^{\prime}(\tau) y(\tau)\right) d \tau \geq S_{s}\left(x\left(t_{f}\right)\right)-S_{s}\left(x\left(t_{0}\right)\right)
$$

Moreover choose $S_{c}(z) \triangleq-z+\beta$ as storage function associated to the controller. This storage function satisfies $S_{c}(z) \geq 0$ since $z \leq \beta$ holds. Using the feedback relations $u=e_{u}-v$ and $w=e_{y}+y$ (see figure 1) we get

$$
\begin{gathered}
S_{s}\left(x\left(t_{0}+\delta\right)\right)+S_{c}\left(z\left(t_{0}+\delta\right)\right) \leq S_{c}\left(z\left(t_{0}\right)\right)+S_{s}\left(x\left(t_{0}\right)\right)+ \\
\int_{t_{0}}^{t_{0}+\delta}\left(e_{u}^{\prime}(\tau) y(\tau)+e_{y}^{\prime}(\tau) u(\tau)-\right. \\
\left.\left.\left.\gamma u^{\prime}(\tau) u(\tau)\right)-\tilde{\rho} y^{\prime}(\tau) y(\tau)\right)\right) d \tau
\end{gathered}
$$

for all $x \in \mathcal{X}_{z_{t_{0}}}$. If system (14) is zero-state detectable and $x_{s}(t) \in \mathcal{X}_{z_{t}}$ for all $t \geq 0$ the inequality (17) guarantees that if $e_{u}(\cdot), e_{y}(\cdot) \in \overline{\mathcal{L}}_{2}$ then $u(\cdot), y(\cdot) \in \mathcal{L}_{2}$. Moreover if $e_{u}(\cdot)=0$ and $e_{y}(\cdot)=0$, since $u(\cdot), y(\cdot) \in \mathcal{L}_{2}$, the zerostate detectability of the system guarantees that

$$
\lim _{t \rightarrow \infty} x_{s}(t)=0 .
$$

Remark 2. Note that, in order to get robustness, is important to know the system's passivity property and the equilibrium pair $u_{s}, y_{s}$.

\section{EXAMPLE}

This example illustrates the position control for a flexible 2-link robot manipulator in the horizontal plane with dynamics

$$
\begin{aligned}
& M(q) \ddot{q}+C(q, \dot{q}) \dot{q}+F(\dot{q})=K(\theta-q) \\
& J \ddot{\theta}+K(\theta-q)=u
\end{aligned}
$$

where $q=\left[q_{1}, q_{2}\right]$ are the generalized configuration coordinates for a system with two degrees of freedom, $\theta=\left[\theta_{1}, \theta_{2}\right]$ are the motor angles, $C(q, \dot{q}) \dot{q}$ describes the centrifugal and Coriolis forces, $F(\dot{q})=\left[\beta_{1} \dot{q}_{1}, \beta_{2} \dot{q}_{2}\right]^{\prime}$ describes the linear viscous friction, $J=\left[\begin{array}{llll}J_{1} & 0 ; & 0 & J_{2}\end{array}\right]$ represents actuator inertia reflected to the links side of the gears, $K=\left[\begin{array}{llll}k_{1} & 0 ; & 0 & k_{2}\end{array}\right]>0$ models the stiffness of the joints and $M(q)$, that brings information on the inertia of the system, is a positive symmetric matrix for all $q$. Under weak assumptions (see Van der Schaft [2000]) the system (18) is passive with supply rate $\dot{\theta}^{\prime} u$ and it is zero-state detectable with respect to $[q, \theta]$. In particular the matrix entries $m_{i, j}(q)$ of $M(q)$ and $c_{i, j}(q, \dot{q})$ of $C(q, \dot{q})$ are

$$
\begin{aligned}
& m_{1,1}(q)=a_{1}+2 a_{3} \cos \left(q_{2}\right)+2 a_{4} \sin \left(q_{2}\right) \\
& m_{1,2}(q)=m_{2,1}(q)=a_{2}+a_{3} \cos \left(q_{2}\right)+a_{4} \sin \left(q_{2}\right) \\
& m_{2,2}(q)=a_{2}, \quad c_{2,2}(q, \dot{q})=0 \\
& c_{1,1}(q, \dot{q})=-h(q) \dot{q_{2}}, \quad c_{2,1}(q, \dot{q})=h(q) \dot{q}_{1} \\
& c_{1,2}(q, \dot{q})=-h(q)\left(\dot{q} \dot{q}_{2}+\dot{q}_{1}\right)
\end{aligned}
$$

where $h(q)=a_{3} \sin \left(q_{2}\right)-a_{4} \cos \left(q_{2}\right), a_{1}=I_{1}+m_{1} l_{c_{1}}^{2}+I_{e}+$ $m_{e} l_{c e}^{2}+m_{e} l_{1}^{2}, a_{2}=I_{e}+m_{e} l_{c e}^{2}, a_{3}=m_{e} l_{1} l_{c e} \cos \left(\delta_{e}\right)$ and $a_{4}=m_{e} l_{1} l_{c e} \sin \left(\delta_{e}\right)$ (see Fig. 2). The model parameters for the nominal model are $m_{1}=1, l_{1}=1, m_{e}=2$, $\delta_{e}=\pi / 6, I_{1}=0.12, l_{c 1}=0.5, I_{e}=0.25, l_{c e}=0.6$, $J_{1}=0.6, J_{2}=0.5, k_{1}=1, k_{2}=1.5, \beta_{1}=0.01$ and $\beta_{2}=0.05$ in the appropriate units of measure. Introducing the state variable $x \triangleq[\theta, \dot{\theta}, q, \dot{q}]$ the state and control constraint sets are

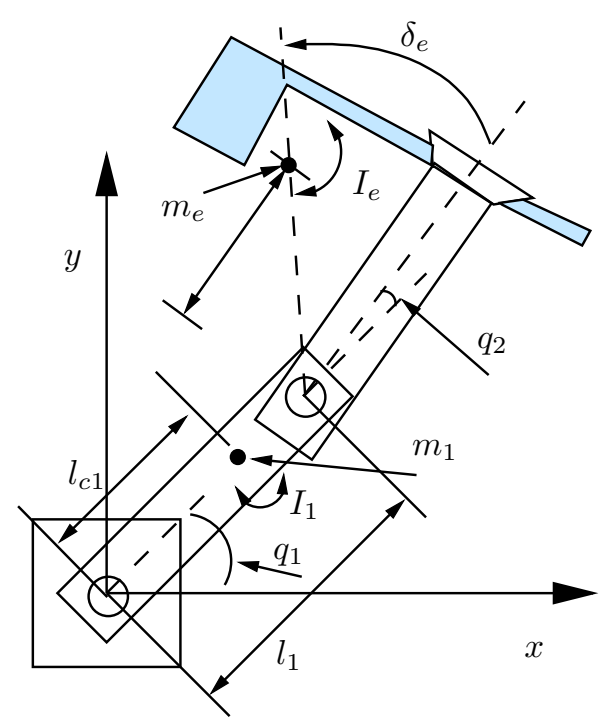

Fig. 2. Two-link arm manipulator

$$
\begin{aligned}
\mathbb{X}= & \left\{x \in \mathbb{R}^{8} \mid \theta_{i}, q_{i} \in[-5 \pi / 6,5 \pi / 6],\right. \\
& \left.\dot{\theta}_{i}, \dot{q}_{i} \in[-50,50], i=1,2\right\}, \\
\mathbb{U}= & \left\{u \in \mathbb{R}^{2} \mid u_{i} \in[-1000,1000], \quad i=1,2\right\},
\end{aligned}
$$

The robot is initially at rest with $x_{0}=\mathbf{0}$ and it is required to reach the steady state $\bar{q}_{1}=\bar{\theta}_{1}=\pi / 3$ and $\bar{q}_{2}=$ $\bar{\theta}_{2}=\pi / 2$ while satisfying the constraints. The discretetime model is implicitly defined via the optimization process; the optimization packages described in Wächter and Biegler [2006], Wyk et al. [2010] were employed for all the simulations. The controller uses $N=1750$ with a prediction horizon $T=3.5 \mathrm{~s}$ corresponding to a sampling period of 0.01 seconds. The selected stage cost is $\ell(x, u)=$ $5000\|q-\bar{q}\|^{2}+5000\|\theta-\bar{\theta}\|^{2}+0.01\|u\|^{2}$.

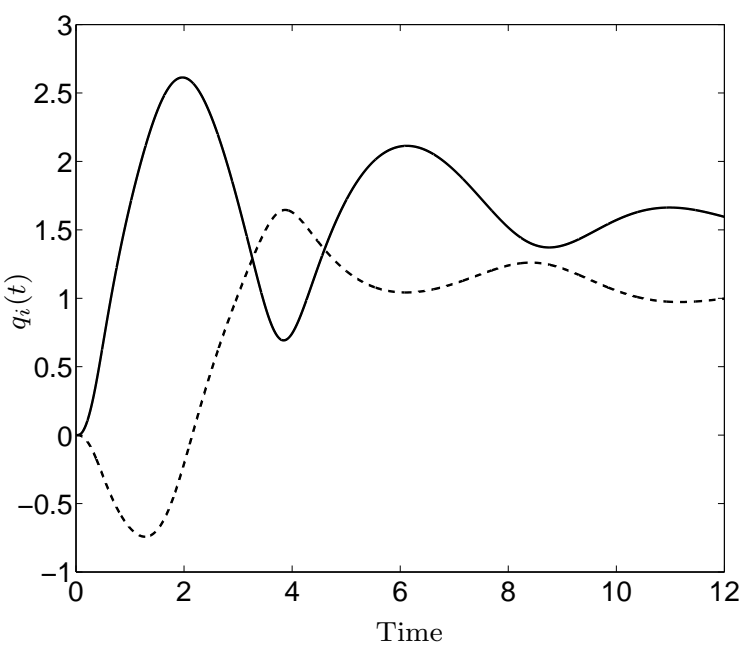

Fig. 3. State variables $q$ vs time: $q_{1}$ (dashed) and $q_{2}$ (solid).

The terminal ingredient has been chosen as $x(T)=$ $[\pi / 3, \pi / 2,0,0, \pi / 3, \pi / 2,0,0]$. Finally the parameters to enforce output strict passivity of the controller have been chosen as $\beta=1000$ and $\gamma=0.01$. The large value for the parameter $\beta$ allows to tolerate consistent discrepancies between the nominal model and the real system whenever 


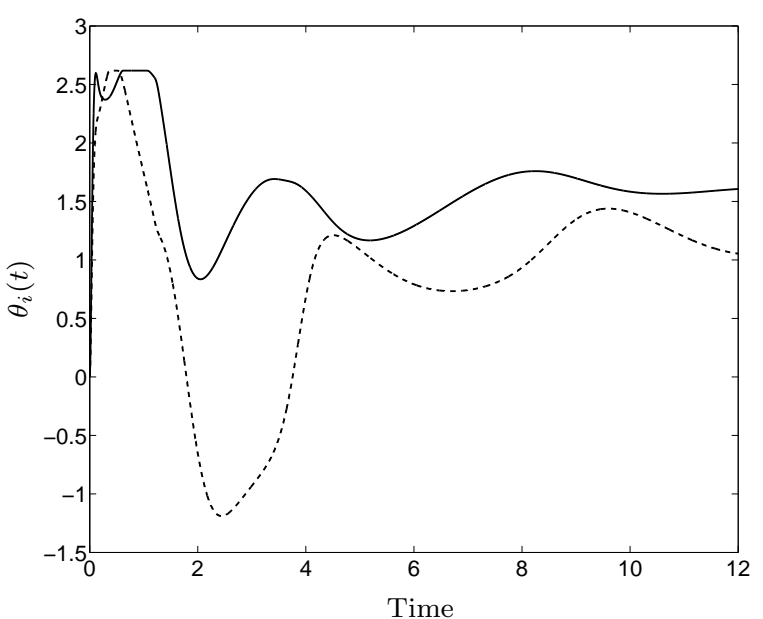

Fig. 4. State variables $\theta$ vs time: $\theta_{1}$ (dashed) and $\theta_{2}$ (solid).

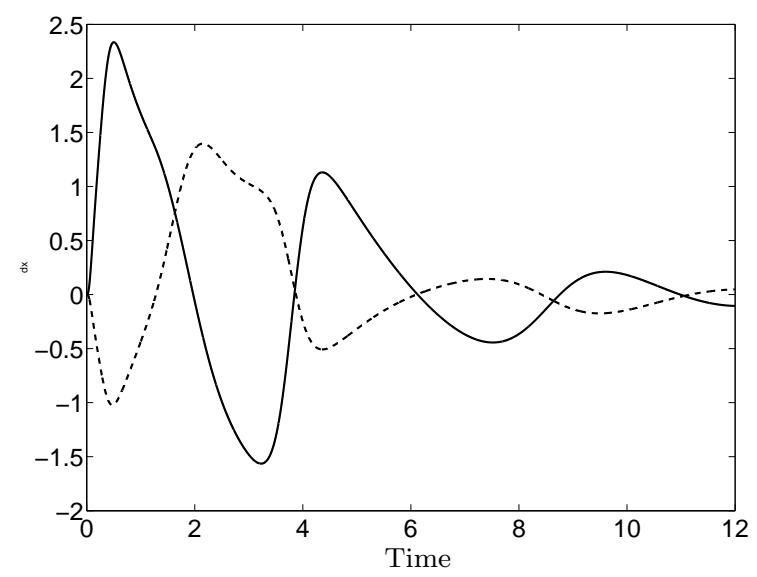

Fig. 5. State variables $\dot{q}$ vs time: $\dot{q}_{1}$ (dashed) and $\dot{q}_{2}$ (solid).

it remains passive. Simulation experiments have been carried out assuming a different load and different values of the coefficient describing the friction and the stiffness. In particular the reported results have been obtained with, $m_{e}=1, \delta_{e}=\pi / 12, I_{e}=0.25, l_{c e}=1, k_{1}=0.4$, $k_{2}=0.6, \beta_{1}=10^{-5}$ and $\beta_{2}=10^{-6}$. It can be seen in Figs. 3 and 4 that the controller is able to drive the robot arm to the desired position while satisfying input and state constraints (see Figs. 5, 6 and 8) despite the lack of the precise knowledge of the model. The constraint $z(t) / \beta \leq 1$ is satisfied as well and it becomes active during the transient.

\section{CONCLUSION}

In this paper an MPC formulation for strictly output passive systems subject to input and state constraints has been proposed with guaranteed robustness properties. The nominal MPC control scheme has been enriched with a dynamic equation allowing to impose strict output passivity of the controller and consequently the passivity of the closed loop system. The scheme has been proved to be robust against model errors preserving passivity of the system to be controlled whenever feasibility of the optimization problem is not lost. The robustness property

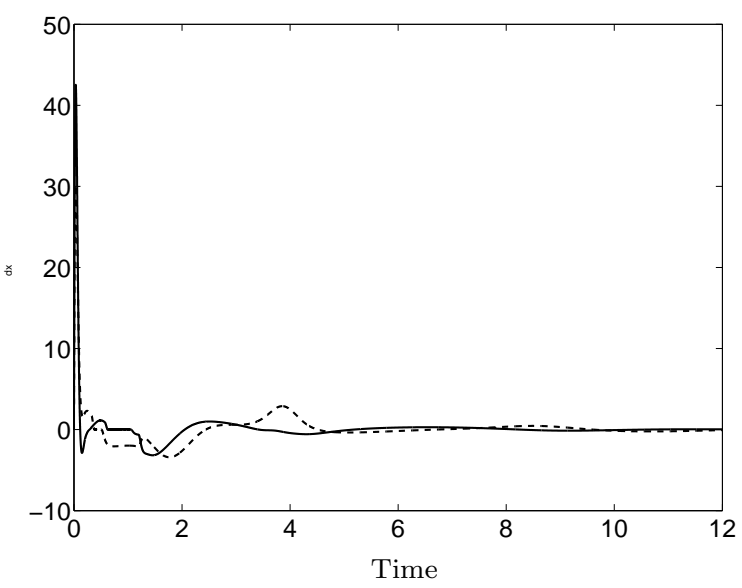

Fig. 6. State variables $\dot{\theta}$ vs time: $\dot{\theta}_{1}$ (dashed) and $\dot{\theta}_{2}$ (solid).

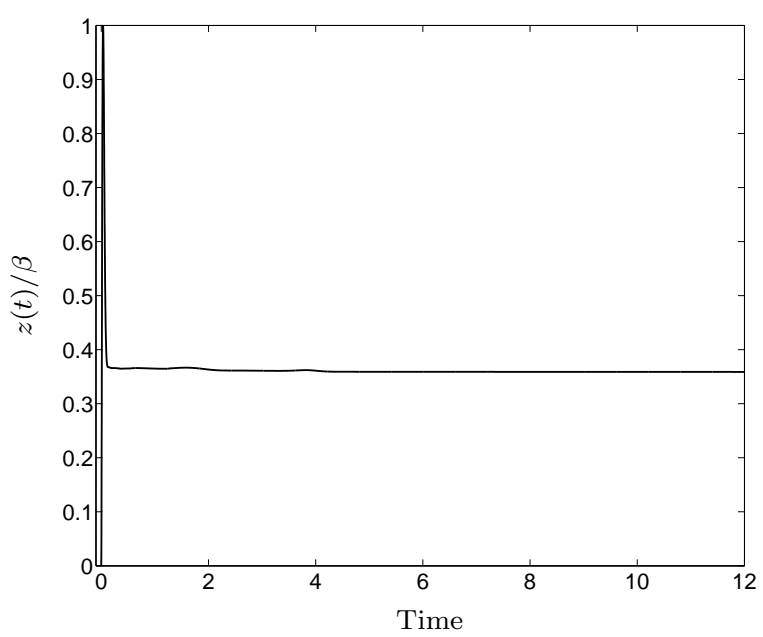

Fig. 7. State variable $z(t) / \beta$ vs time.

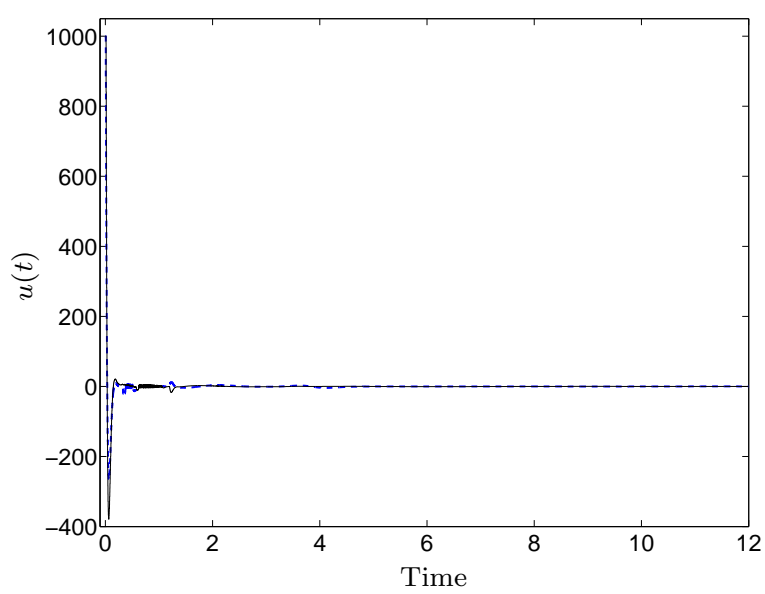

Fig. 8. Input variable $u(t)$ vs time: $u_{1}$ (dashed) and $u_{2}$ (solid).

of the scheme has been illustrated by simulation results concerning the position control problem for a flexible 2-link robot manipulator in the horizontal plane. The convergence to the desired target set is obtained despite 
large mismatches between the nominal model and the real one.

\section{ACKNOWLEDGEMENTS}

This work was supported by EPSRC.

\section{REFERENCES}

D. Angeli, R. Amrit, and J B. Rawlings. On Average Performance and Stability of Economic Model Predictive Control. IEEE Transactions on Automatic Control, 57: 1615-1626, 2012.

D. Limon, T. Alamo, D. M. Raimondo, J. M. Bravo, D. Munoz de la Peña, A. Ferramosca, and E. F. Camacho. Input-to-State Stability: an unifying framework for Robust Model Predictive Control. In L. Magni, D. M. Raimondo, and F. Allgöwer, editors, Nonlinear Model Predictive Control: Towards New Challenging Applications, pages 1-26. Springer-Verlag, New York, 2009.

D. Q. Mayne, J. B. Rawlings, C. V. Rao, and P. O. M. Scokaert. Constrained model predictive control: stability and optimality. Automatica, 36:789-814, June 2000. Survey paper.

R. Ortega and M. Spong. Adaptive motion control of rigid robots: A tutorial. Automatica, 25:877-888, 1989.

R. Ortega, A. Loria, P. J. Nicklasson, and H. Sira-Ramrez. Passivity-based Control of Euler-Lagrange Systems: Mechanical, Electrical and Electromechanical Applications. Springer-Verlag, London, 1998.

J. A. Primbs, V. Nevistić, and J. C. Doyle. A receding horizon generalization of pointwise min-norm controllers. IEEE Transactions on Automatic Control, 45:898-909, 2000.

S. J. Qin and T. A. Badgwell. A survey of industrial model predictive control technology. Control Engineering Practice, 11:733-764, 2003.

M. Janković R. Sepulchre and P. Kokotović. Constructive Nonlinear Control. Springer, 1997.

T. Raff, C. Ebenbauer, and F. Allgöewer. Assessment and Future Directions of Nonlinear Model Predictive Control, chapter Nonlinear model predictive control: A passivity-based approach, pages 151-162. Lecture Notes in Control and Information Sciences. Springer, 2007.

James B. Rawlings and David Q. Mayne. Model Predictive Control: Theory and Design. Nob Hill, Madison, Wisconsin, August 2009.

A.R. Teel, T.T. Georgiou, L. Praly, and E.D. Sontag. Input-output stability. In W. S. Levine, editor, The Control Systems Handbook: Control System Advanced Methods, Second Edition, pages 44.1-44.23 (1011-1033). CRC Press, Boca Raton, 2010.

A.J. Van der Schaft. $L_{2}$-Gain and Passivity Techniques in Nonlinear Control, 2nd Edition. Springer-Verlag, London, 2000.

A. Wächter and L. T. Biegler. On the implementation of a primal-dual interior point filter line search algorithm for large-scale nonlinear programming. Mathematical Programming, 106(1):25-57, 2006. http://www.coinor.org/Ipopt/.

E. J. Van Wyk, P. Falugi, and E. C. Kerrigan. Imperial College London Optimal Control Software (ICLOCS). Solves nonlinear optimal control problems with constraints, http://www.ee.ic.ac.uk/ICLOCS, 2010.
H. Yan, F. Zhu, M. Xia, and P. J. Antsaklis. Robust stabilizing output feedback nonlinear model predictive control by using passivity and dissipativity. In Proc. of the European Control Conference, pages 2050-2055, 2013. 